\title{
Electronic health records and patient registries in medical oncology departments in Spain
}

\author{
N. Ribelles ${ }^{1}$ (D) - A. Alvarez-Lopez ${ }^{2}$ - A. Arcusa ${ }^{3}$. J. I. Chacon ${ }^{4}$. J. de la Haba ${ }^{5}$. J. García-Corbacho ${ }^{6}$. J. Garcia-Mata ${ }^{7}$. \\ C. Jara ${ }^{8} \cdot$ J. M. Jerez ${ }^{9} \cdot$ M. Lázaro-Quintela ${ }^{10} \cdot$ L. Leon-Mateos ${ }^{11} \cdot$ N. Ramirez-Merino ${ }^{12} \cdot$ A. Tibau $^{13} \cdot$ A. Garcia-Palomo ${ }^{14}$
}

Received: 5 February 2021 / Accepted: 30 March 2021 / Published online: 17 April 2021

(c) The Author(s) 2021

\begin{abstract}
Purpose We aimed to evaluate the current situation of electronic health records (EHRs) and patient registries in the oncology departments of hospitals in Spain.

Methods This was a cross-sectional study conducted from December 2018 to September 2019. The survey was designed ad hoc by the Outcomes Evaluation and Clinical Practice Section of the Spanish Society of Medical Oncology (SEOM) and was distributed to all head of medical oncology department members of SEOM.

Results We invited 148 heads of oncology departments, and 81 (54.7\%) questionnaires were completed, with representation from all 17 Spanish autonomous communities. Seventy-seven (95\%) of the respondents had EHRs implemented at their hospitals; of them, over $80 \%$ considered EHRs to have a positive impact on work organization and clinical practice, and $73 \%$ considered that EHRs improve the quality of patient care. In contrast, $27(35.1 \%)$ of these respondents felt that EHRs worsened the physician-patient relationship and conveyed an additional workload $(n=29 ; 37.6 \%)$. Several drawbacks in the implementation of EHRs were identified, including the limited inclusion of information on both outpatients and inpatients, information recorded in free text data fields, and the availability of specific informed consent. Forty-six (56.7\%) respondents had patient registries where they recorded information from all patients seen in the department.

Conclusion Our study indicates that EHRs are almost universally implemented in the hospitals surveyed and are considered to have a positive impact on work organization and clinical practice. However, EHRs currently have several drawbacks that limit their use for investigational purposes.
\end{abstract}

Clinical trial registration Not applicable

Keywords Electronic health records $\cdot$ Patient registries $\cdot$ Medical oncology $\cdot$ Work organization $\cdot$ Clinical practice $\cdot$ Clinical research

\section{Introduction}

According to the International Organization for Standardization (ISO) [1], an electronic health record (EHR) is "a repository of patient data in digital form, stored and exchanged securely, and accessible by multiple authorized users. It contains retrospective, concurrent, and prospective information, and its primary purpose is to support continuing, efficient and quality integrated health care". A previous systematic review has shown that EHRs improve the quality of health care by reducing documentation time, increasing guideline

N. Ribelles

nuria.ribelles.sspa@juntadeandalucia.es

Extended author information available on the last page of the article adherence, and reducing medication errors and drug-related adverse events [2]. Although the implementation of EHRs has not been shown to reduce mortality [2,3], some studies have demonstrated that they can moderately improve morbidity outcomes [3].

EHRs may also be an important tool for clinical research. Within the oncology field, they have been used for evaluating the cancer risk associated with some treatments $[4,5]$ and to create predictive models $[6,7]$ that allow personalized care and to evaluate the quality of cancer care [8], to cite some examples. In clinical trials, EHRs facilitate accessibility to medical records and thus speed up the identification of potential participants $[9,10]$. They also facilitate the capture of electronic data in an automated way [11], helping to monitor the review of source 
data, such as EHR, and thus avoid difficulties in interpreting the handwritten characters of doctors. In addition, the availability of electronic tools to find specific words in long text and even potentially allow remote monitoring, which recently became a major issue during the pandemic, is useful. Additionally, EHRs can be a source for selecting patients treated with a contemporary standard of care as an external control to interpret the efficacy observed in early phase single-group clinical trials [12]. Early in drug development, EHRs may be used to identify potential candidates for the treatment of cancer by analyzing the effects on survival of noncancer drugs in patients with cancer-so-called drug repurposing [13, 14]. EHRs are also an important source for obtaining real-world evidence for the effects of interventions [15]. In this regard, the American Society of Clinical Oncology in collaboration with the MITRE Corporation (a private, not-for-profit company providing engineering and technical guidance for the federal government in the US) launched an initiative in 2019, the mCODE (Minimal Common Oncology Data Elements), for establishing a core set of structured data elements for oncology EHRs that would enable the treatment of every cancer patient to contribute to comparative effectiveness analysis $[16,17]$.

There is no information on the availability and characteristics of EHRs in oncology at the country level in Spain. Therefore, the Outcomes Evaluation and Clinical Practice Section of the Spanish Society of Medical Oncology (SEOM) conducted this survey with the aim of evaluating the degree of implementation of EHRs by the medical oncology departments of hospitals in Spain, describing the situation regarding cancer registries among those departments, and assessing their willingness to share patient data.

\section{Subjects and methods}

\section{Study design}

This was a cross-sectional study that was undertaken from December 2018 to September 2019. Since the survey was addressed to health professionals and no individual patient datum was recorded, evaluation by the ethics committee was not required. Participants were informed of the objectives of the survey and that their participation was not completely anonymous. That is, centers were identified in the questionnaire, but the health professional reporting the information was not. They were also informed that the results of the survey would be reported in an aggregate manner.

\section{The survey}

The survey was designed ad hoc by 11 members (NR, AGP, IAL, AAL, JGM, JHR, CJS, MLQ, LLM, NRM and ATM) of the Outcomes Evaluation and Clinical Practice Section of the SEOM, which in turn supported this study. The survey was distributed by email to all SEOM members with the category of head of medical oncology department with a cover letter explaining the objectives of the study. Three reminders were sent via email. Finally, the members of the Executive Committee of this section of SEOM tried to contact the nonresponders directly via email or phone.

The surveyed comprised 17 questions that could be grouped into the following sections:

1. Practice identification and age, position and experience of the health professional who filled out the questionnaire (1 question).

2. Data on the availability of patient registries in the department, type of registries, and need for a national cancer registry (3 questions).

3. Data on the availability of an electronic prescription system and its characteristics (2 questions).

4. Data on EHRs (11 questions):

a. EHR availability

b. Length of time that EHR has been available

c. It is considered of interest in implementing EHR in the department if not available

d. What are the characteristics of the information included in EHRs?

e. In which format the data are recorded

f. Degree of agreement on the impact of the availability of an EHR on several issues (measures on a 3-point Likert scale)

g. Availability of tools for extracting data from EHR

h. Possibility of accessing diagnostic tests through the EHR

i. Compatibility of the EHR for use in clinical trials

j. Availability of informed consent for using patient information from the EHR

k. Willingness to share information from the EHRs with other investigators.

The complete questionnaire is available as Supplementary Information, both in the English and Spanish versions.

\section{Statistical analysis}

We used a convenience sample; therefore, we did not perform any sample size calculations. 
Our data are essentially descriptive. We used absolute and relative frequencies to present qualitative variables.

All analyses were performed using Microsoft Excel 365.

\section{Results}

\section{Response rate and characteristics of the respondents}

We invited 148 heads of oncology departments throughout Spain, and after reminders, $81(54.7 \%)$ answered the questionnaires. All 17 Spanish autonomous communities (AACC) and 1 of the 2 Spanish autonomous cities were represented by at least one hospital. However, hospitals from 5 AACCs represented $64.1 \%$ of the responses: Catalonia $(n=14,17.5 \%)$, Madrid $(n=11,13.6 \%)$, Andalusia $(n=11$, $13.6 \%)$, Valencia $(n=9,11.1 \%)$, and Galicia $(n=7,8.6 \%)$ (Table 1). Questionnaires were completed by the head of the department $(n=54,66.6 \%)$, followed by the head of section $(n=21,25.9 \%)$ and the senior registrar $(n=6,7.4 \%)$. Seventy-three $(90.1 \%)$ of the respondents were older than 45 years, and $66(81.4 \%)$ had professional experience greater than 20 years.

\section{Availability and characteristics of patient registries}

Forty-six $(56.7 \%)$ of the centers had a register or database where the information of all patients attending the clinical offices was recorded. There were no relevant differences in

Table 1 Number of hospitals with answered surveys

\begin{tabular}{ll}
\hline $\begin{array}{l}\text { Spanish autono- } \\
\text { mous communities }\end{array}$ & $N$ \\
\hline Andalusia & $11(13.5 \%)$ \\
Aragon & $3(3.7 \%)$ \\
Asturias & $1(1.2 \%)$ \\
Balearic Islands & $2(2.4 \%)$ \\
Basque Country & $3(3.7 \%)$ \\
Canary Islands & $1(1.2 \%)$ \\
Cantabria & $1(1.2 \%)$ \\
Castile and Lion & $4(4.9 \%)$ \\
Castilla La Mancha & $4(4.9 \%)$ \\
Catalonia & $14(17.3 \%)$ \\
Ceuta & $1(1.2 \%)$ \\
Estremadura & $3(3.7 \%)$ \\
Galicia & $7(8.6 \%)$ \\
Madrid & $11(13.5 \%)$ \\
Murcia & $3(3.7 \%)$ \\
Navarre & $1(1.2 \%)$ \\
Rioja & $2(2.4 \%)$ \\
Valencia & $9(11.1 \%)$ \\
\hline
\end{tabular}

this regard among the AACCs with the largest number of participant centers, except for Valencia, who reported that only 3 out of the 9 (33.3\%) participant hospitals had a patient registry/database (Fig. 1). There were only five AACCs in which $100 \%$ of the responders registered all patients treated in their services (Asturias, Castilla-León, Ceuta, La Rioja and Navarra). Additionally, 22 (27.1\%) respondents reported the presence of other registries conducted by some of the medical staff of the department. The information recorded in a patient registry/database was the type of cancer $(n=73$, $90.1 \%)$, stage $(n=55,67.9 \%)$, date of diagnosis $(n=51$, $62.9 \%)$, type of treatment received $(n=46,56.7 \%)$ and date and clinical status at the last follow-up visit $(n=27,33.3 \%)$.

All but 2 of the respondents (97.5\%) considered that there was a need to create a national registry of cancer patients.

\section{Availability of an electronic prescription system and its characteristics}

Seventy-six out of the 81 participants $(93.8 \%)$ reported having an electronic prescription system. Of those with the electronic prescription system, $42(55.2 \%)$ had direct access to the electronic prescription system from the EHR, 33 (43.4\%) could directly obtain information about the prescribed treatments, 30 (39.4\%) could request that information through the Pharmacy Department and 3 (3.9\%) could not obtain that information.

\section{Electronic health record}

Seventy-seven (95.1\%) of the respondents had EHRs implemented at their hospitals, and of them, 63 (81.8\%) had used that tool for a period longer than 5 years. All four respondents without an EHR implemented at their center considered that, currently, the EHR is an essential tool.

In over $90.9 \%$ of cases, the EHR was designed and maintained by the AACC Health Service, but only one-third had specific templates for recording information (Fig. 2). In $57.1 \%$ of the centers, the EHR includes information on outpatient consultation, as well as on hospitalized patients. Data from the EHR are recorded in a structured manner (i.e., using drop-down lists) in $10.4 \%$ of the centers, while $48.1 \%$ of them use free text data fields, and $41.5 \%$ combine the two systems.

In the survey, several questions were included to determine opinions about different aspects related to the use of EHRs. More than eighty percent considered that its use improves access to patient information $(68 ; 88.3 \%)$, that it is essential to analyze health outcomes $(66 ; 85.7 \%)$ and that it favors uniformity of work by all physicians $(64 ; 83.1 \%)$. Between seventy and eighty percent supported the value of EHR as an essential source of information to improve knowledge (61; $79.2 \%)$ and to progress in our work system (58; 75.3\%). In 


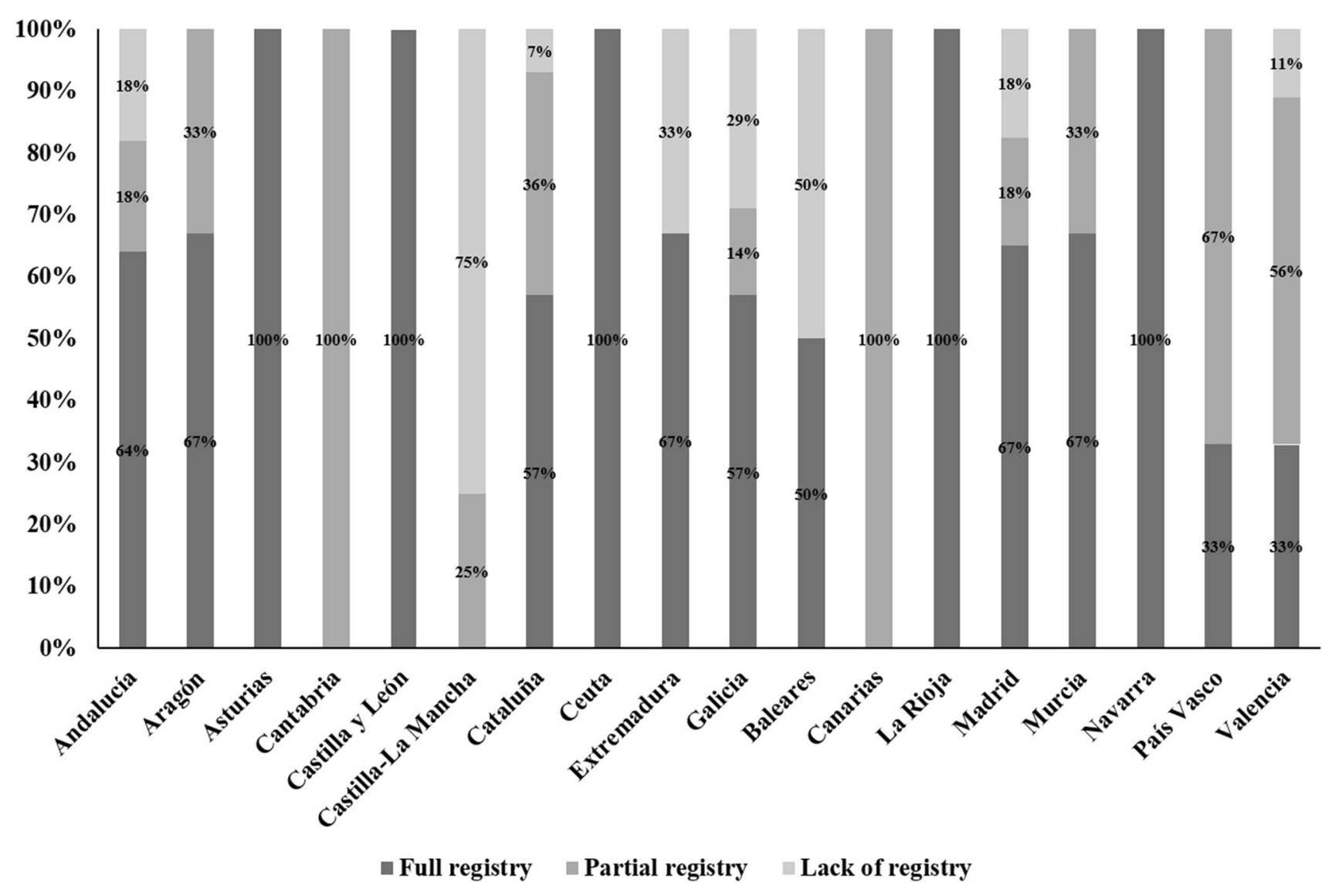

Fig. 1 Availability of patient registries by autonomous communities

addition, the use of EHRs allows us to easily obtain updated information about patient status $(58 ; 75.3 \%)$ and contributes to improving the quality of patient care (56: 72.7\%). However, there was no such clear agreement on whether use of the EHR worsened the physician-patient relationship (yes $27 ; 35.1 \%$ vs no $31 ; 40.2 \%$ ) or whether it involved an additional workload (yes $29 ; 37.6 \%$ vs no $34 ; 44.1 \%$ ) (Fig. 3 ).

Regarding extraction of data from the EHRs (Fig. 4), 24 (31.2\%) respondents reported that they could not extract information. Only $9(11.7 \%)$ of the respondents could directly extract information from the EHRs, and 23 (29.8\%) had to request the information from those responsible for the EHRs within the AACC Health Service. Only 32 (41.5\%) respondents could directly access information from diagnostic tests from the EHR. Forty-eight (62.3\%) respondents thought that their EHR met the needed features to be considered a proper EHR by the sponsors of clinical trials. Thirtyfive respondents (45.4\%) reported that their patients signed a general informed consent form for the administration of treatments in which the use of their data collected in the EHR was also authorized. Moreover, sixteen other respondents $(20.8 \%)$ pointed out that their patients signed a specific consent form to authorize the use of their data.
Overall, nearly $85 \%$ of the respondents were willing to share data from EHRs within the frame of a project sponsored by SEOM and considered that sharing data might have a positive impact on obtaining information on health outcomes from cancer patients and would allow one to obtain information on the prevalence and incidence of cancer (Fig. 5).

\section{Discussion}

Our study indicates that EHRs are almost universally implemented in the hospitals surveyed and are considered to have a positive impact on work organization and clinical practice. However, there are several drawbacks to how EHRs are implemented that limit optimal use for both clinical practice and clinical research.

According to our survey, $95.1 \%$ of the participating hospitals had implemented an EHR, a figure that is entirely consistent with the $93.3 \%$ coverage of the National Health System Electronic Health Records Project reported by the Spanish Ministry of Health as stated in the last report of October 2020 [18]. Those hospitals where an EHR is not yet 


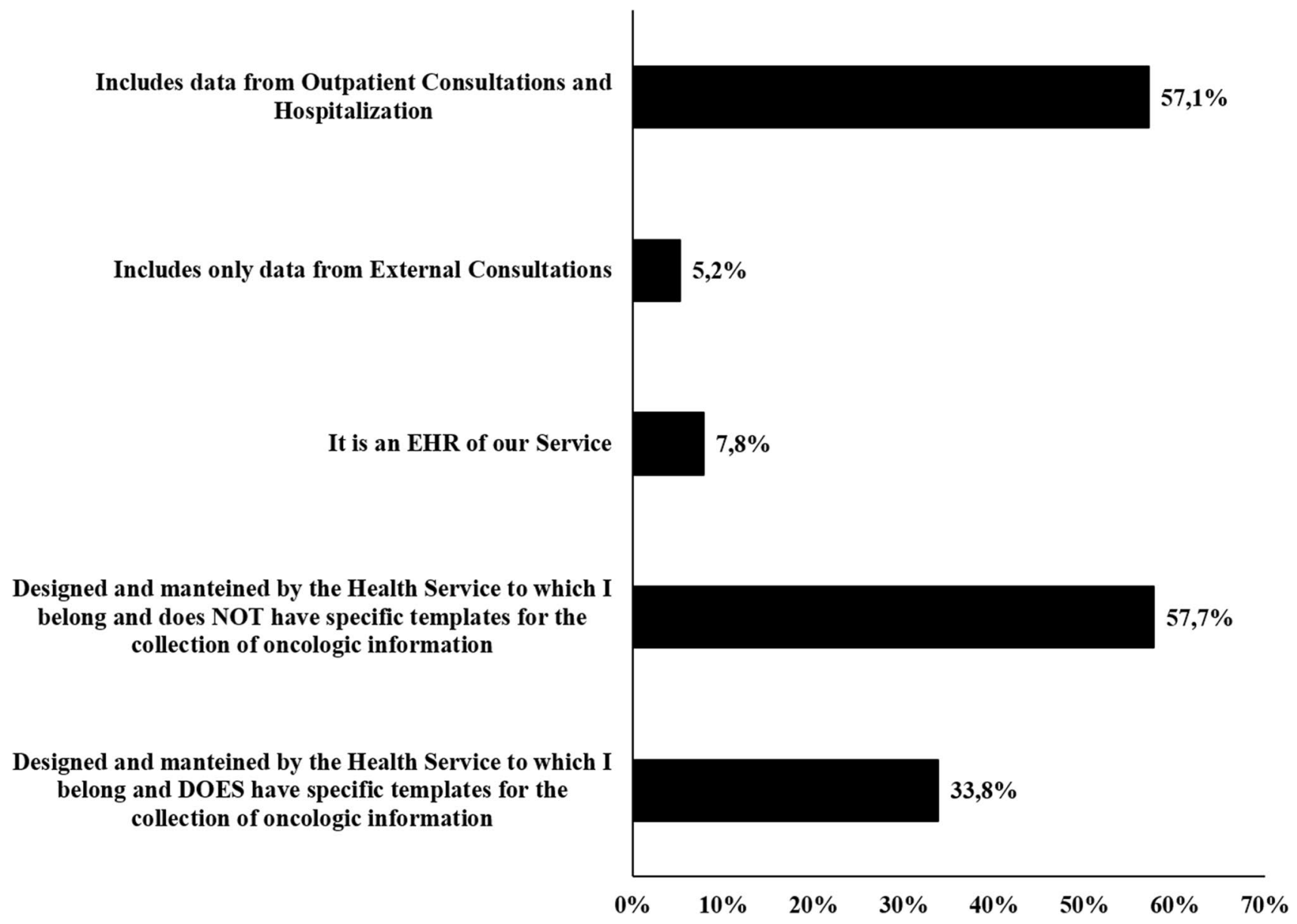

Fig. 2 Characteristics of the electronic health record

available considered it an essential tool. Most respondents believe that the availability of EHRs has a positive impact on work organization and clinical practice and agree that it improves the quality of patient care. However, it is important to note that $35.1 \%$ of surveyed oncologists think that the EHR may interfere with the doctor-patient relationship. This concern is somehow justified, as the use of EHRs may disrupt communication (e.g., interrupted speech), and this perception of a negative impact on the patient-doctor relationship has been reported previously [19]. However, a systematic review found that EHRs produce no change in patient satisfaction or patient-doctor communication [20]. Our study also shows that $37.6 \%$ of the centers surveyed consider that EHRs increase workload. Studies conducted in primary care show that, in fact, the implementation of EHRs can improve productivity/efficiency in physician workloads [21]. Improving the usability of the EHR may decrease this perception of increased workload [22].

Although two-thirds of respondents reported that their EHRs meet the needed characteristics for being considered a proper EHR by sponsors of clinical trials, in our view, there are still many drawbacks in the implementation of EHRs in Spain that limit their use for research purposes, including clinical trials. Only $57.1 \%$ of centers have an EHR that includes information on both outpatients and inpatients. Almost half of centers use an EHR where the information is recorded in free text data fields. Approximately two-thirds of respondents either cannot extract information from the EHR or must request the desired information from the person responsible for this system at the AACC Health System.

We did not include questions in our survey regarding the specific content of the EHR. The ASCO project mCODE recommends core elements be included for cancer research treatment, including patient information (demographics, comorbid conditions and performance status), cancer characteristics, genomics information, laboratory and vital sign information, including tumor markers, cancer treatment information, and cancer disease status [16]. We think this information could only be obtained in our setting by designing and implementing the appropriate tools that allow us to extract and analyze this information from the content of EHRs. Fortunately, over $80 \%$ of 


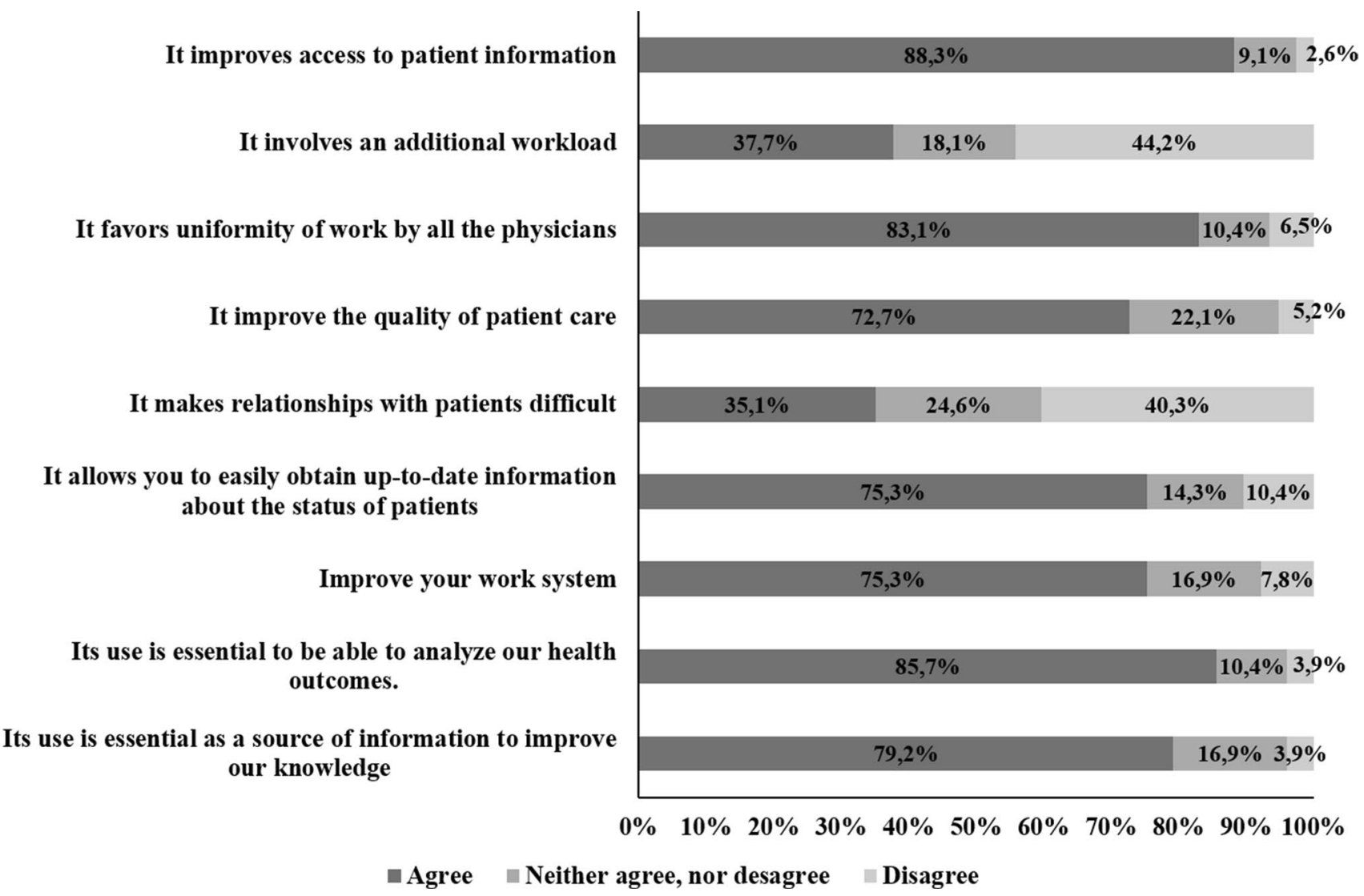

Fig. 3 Impact of the electronic health record

respondents were willing to share their data, but this could be hampered by the fact that it is not possible to share the data contained in the EHRs from the health systems from different AACCs. Therefore, by involving additional stakeholders (i.e., pharmacists, hospital management, and scientific societies, such as SEOM, among others), similar projects could be implemented in Spain in the future.

Nearly $84 \%$ of the centers surveyed had some type of patient register or database, and the registry included information on all patients attended in $56.7 \%$ of the surveyed hospitals. It is possible that this high number of centers with registries was facilitated by the high use of EHRs. In the United States, it has been reported that the availability of EHRs facilitates the development and implementation of patient registries [23]. The information included in the registries from our survey is limited, especially for evaluating treatment outcomes, since only $56.7 \%$ recorded the type of treatment received, and only $33.3 \%$ recorded the clinical status at the last follow-up. This situation is further complicated by the fact that despite the availability of an electronic prescription system in $93.8 \%$ of centers, $43.3 \%$ could not directly obtain information on prescribed treatments.

A limitation of our survey is the relatively low rate of participants, since only $54.7 \%$ of the hospitals that were contacted answered the questions proposed. Therefore, despite the involvement of centers from all except one administrative territory of Spain, our results cannot be considered representative of the situation at a national level. Nevertheless, the high percentages of agreement obtained in certain responses supports the representativeness of our results. It is also possible that the participants in our survey were those more interested in the use of EHRs, and their opinion could be biased to a positive perception of the usefulness of EHRs. 
I don't know if I can extract information from the EHR

I cannot extract information from the EHR

I have to request the information from those responsible for the EHR at my Health Service

I can extract the information directly

Yes, I can obtain information from the data collected both in the free text fields and in the structured ones

Yes, but only from data collected in a structured way $0 \%$

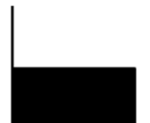

$5,20 \%$

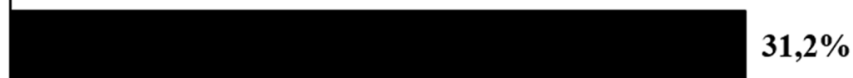

$31,2 \%$

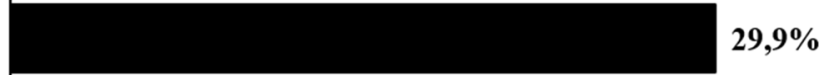

$29,9 \%$

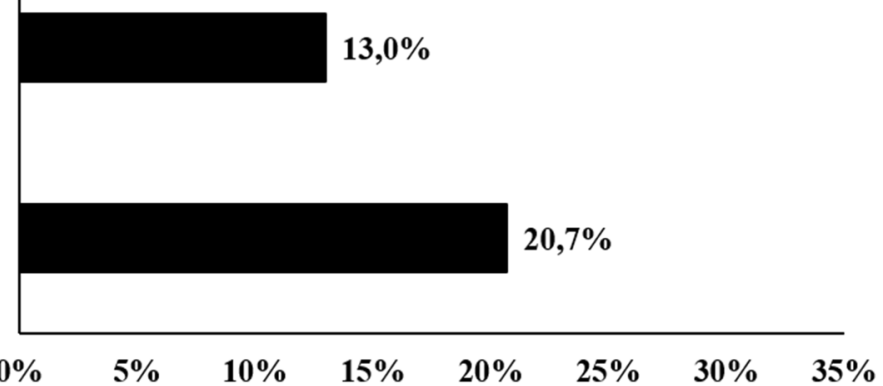

Fig. 4 Information extraction from the electronic health record

However, the importance of EHRs in oncology research is indubitable [23, 24], especially with the increasing relevance of real-world data [25].

According to data obtained by our national survey, the implementation and use of EHRs in Spain appears to have a wide and extensive distribution. However, the EHR's reliance on seventeen different healthcare systems results in several notable weaknesses. Information systems are not compatible with each other and do not always collect the same data. Therefore, it is not possible in any way to obtain information for joint analysis of large-scale data or to compare health outcomes between hospitals or autonomous communities. Furthermore, in most EHRs, it is not possible to obtain or analyze valuable health outcomes.

On the other hand, the use of patient registries is still less implemented. Not all medical oncology departments have registries of the patients they attend, and if they do, they do not always include all their patients or collect enough data. In this sense, it should be highlighted that practically all of the oncologists surveyed consider it necessary to create a National Cancer Registry like those available in most countries of the same level.

The development and implementation of a National Cancer Registry first and a national EHR in the second should be an objective of the health authorities, using consensus as a way to establish what collected data is considered essential. Of course, a national oncology information system of these characteristics should have the appropriate tools to extract data and health outcomes. There is no doubt that explicit support and legal coverage from health authorities are essential for the implementation of such an information system. However, the technology necessary for its development could come from other collaborative sources of the academic and university environment.

Finally, it cannot be ignored that analysis of the data collected in an information system of such characteristics would allow planning the distribution of available resources for the care of cancer patients in the most efficient way. 
I do not believe that is neccesary to share the clinical data of my patiens

I would be interested in sharing the information of my patients within projects sponsored by SEOM with preestablished criteria by an SOP designed with this purpose that guarantees the value and merits of the data provided by each Service

I think it could improve the selection of patients elegible for clinical trials

I think it could help to obtain therapeutic safety data

I think it would be essential to know more precisely the results of the treatments in our patients

I think it would be of great interest to know incidence and prevalence data
$0,0 \%$
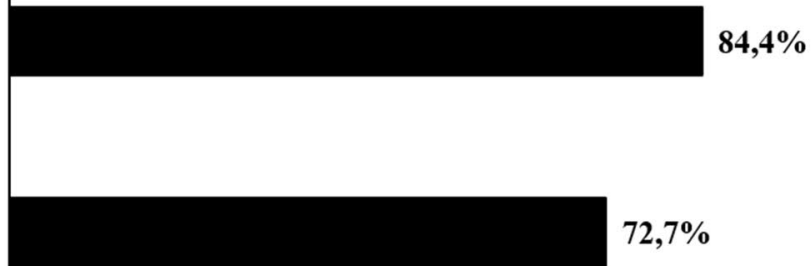

$72,7 \%$

$\begin{array}{llllllllllll}0 \% & 10 \% & 20 \% & 30 \% & 40 \% & 50 \% & 60 \% & 70 \% & 80 \% & 90 \% & 100 \%\end{array}$

Fig. 5 Opinion on sharing patient data from EHRs

Supplementary Information The online version contains supplementary material available at https://doi.org/10.1007/s12094-021-02614-9.

Acknowledgements The authors acknowledge the interest and effort of the participants in answering the survey questionnaire. The authors would like to thank Fernando Rico-Villademoros (COCIENTE SL, Madrid, Spain) for his editorial assistance in the preparation of this manuscript; his participation has been funded by the Spanish Society of Medical Oncology. The authors sincerely appreciate the participation of all medical oncologists who answered the survey.

Author contributions Study concepts: NR, AGP; study design: NR, IAL, AA, JH, JGM, CJ, ML-Q, LL-M, NR-M, AT, AG-P; data acquisition: NR, IAL, AA, JH, JGM, CJ, ML-Q, LL-M, NR-M, AT, AG-P; data analysis and interpretation: NR, IAL, AA, JH, JGM, CJ, ML-Q, LL-M, NR-M, AT, AG-P; statistical analysis: NR, IAL, AA, JH, JGM, CJ, ML-Q, LL-M, NR-M, AT, AG-P; manuscript preparation:NR, IAL, JG-C, LL-M, AT, AG-P; manuscript review: NR, IAL, AA, JICH, JH, JG-C, JG-M, CG, JMJ, ML-Q, LL-M, NR-M, AT, AG-P.

Funding This research was funded by the Spanish Society of Medical Oncology.

\section{Declarations}

Conflict of interest The authors reported no conflict of interest in relation to this manuscript.

Ethics approval This study did not involve the participation of patients; therefore, according to local regulations, ethics approval was not required.

Informed consent This study did not involve the participation of patients; therefore, informed consent was not required.

Open Access This article is licensed under a Creative Commons Attribution 4.0 International License, which permits use, sharing, adaptation, distribution and reproduction in any medium or format, as long as you give appropriate credit to the original author(s) and the source, provide a link to the Creative Commons licence, and indicate if changes were made. The images or other third party material in this article are included in the article's Creative Commons licence, unless indicated otherwise in a credit line to the material. If material is not included in the article's Creative Commons licence and your intended use is not permitted by statutory regulation or exceeds the permitted use, you will 
need to obtain permission directly from the copyright holder. To view a copy of this licence, visit http://creativecommons.org/licenses/by/4.0/.

\section{References}

1. ISO/TC 215 Health informatics (2005-10) ISO/TR 20514:2005 Health informatics-Electronic health record-Definition, scope and context. https://www.iso.org/standard/39525.html. Accessed 16 Oct 2020

2. Campanella P, Lovato E, Marone C, Fallacara L, Mancuso A, Ricciardi W, Specchia ML. The impact of electronic health records on healthcare quality: a systematic review and meta-analysis. Eur J Public Health. 2016;26:60-4.

3. Moja L, Kwag KH, Lytras T, Bertizzolo L, Brandt L, Pecoraro V, Rigon G, Vaona A, Ruggiero F, Mangia M, Iorio A, Kunnamo I, Bonovas S. Effectiveness of computerized decision support systems linked to electronic health records: a systematic review and meta-analysis. Am J Public Health. 2014;104:e12-22.

4. Ibáñez-Sanz G, Guinó E, Pontes C, Morros R, de la Peña-Negro LC, Quijada-Manuitt M, Moreno V. Risk of colorectal cancer in users of bisphosphonates: analysis of population-based electronic health records. Eur J Epidemiol. 2020;35:37-48.

5. Ibáñez-Sanz G, Guinó E, Pontes C, Quijada-Manuitt M, de la Peña-Negro LC, Aragón M, Domínguez M, Rodríguez-Alonso L, Blasco A, García-Rodríguez A, Morros R, Moreno V. Statin use and the risk of colorectal cancer in a population-based electronic health records study. Sci Rep. 2019;9:13560.

6. Wang X, Zhang Y, Hao S, Zheng L, Liao J, Ye C, Xia M, Wang O, Liu M, Weng CH, Duong SQ, Jin B, Alfreds ST, Stearns F, Kanov L, Sylvester KG, Widen E, McElhinney DB, Ling XB. Prediction of the 1-year risk of incident lung cancer: prospective study using electronic health records from the state of maine. J Med Internet Res. 2019;21:e13260.

7. Wu Y, Fan J, Peissig P, Berg R, Tafti AP, Yin J, Yuan M, Page D, Cox J, Burnside ES. Quantifying predictive capability of electronic health records for the most harmful breast cancer. Proc SPIE Int Soc Opt Eng. 2018;10577:105770J.

8. Caldarella A, Amunni G, Angiolini C, Crocetti E, Di Costanzo F, Di Leo A, Giusti F, Pegna AL, Mantellini P, Luzzatto L, Paci E. Feasibility of evaluating quality cancer care using registry data and electronic health records: a population-based study. Int J Qual Health Care. 2012;24:411-8.

9. Weng C, Bigger JT, Busacca L, Wilcox A, Getaneh A. Comparing the effectiveness of a clinical registry and a clinical data warehouse for supporting clinical trial recruitment: a case study. AMIA Annu Symp Proc. 2010;2010:867-71.

10. Visweswaran S, Becich MJ, D'Itri VS, Sendro ER, MacFadden D, Anderson NR, Allen KA, Ranganathan D, Murphy SN, Morrato EH, Pincus HA, Toto R, Firestein GS, Nadler LM, Reis SE. Accrual to clinical trials (ACT): a clinical and translational science award consortium network. JAMIA Open. 2018;1:147-52.

11. Zong N, Wen A, Stone DJ, Sharma DK, Wang C, Yu Y, Liu H, Shi Q, Jiang G. Developing an FHIR-based computational pipeline for automatic population of case report forms for colorectal cancer clinical trials using electronic health records. JCO Clin Cancer Inform. 2020;4:201-9.
12. Carrigan G, Whipple S, Capra WB, Taylor MD, Brown JS, Lu M, Arnieri B, Copping R, Rothman KJ. Using electronic health records to derive control arms for early phase single-arm lung cancer trials: proof-of-concept in randomized controlled trials. Clin Pharmacol Ther. 2020;107:369-77.

13. Wu Y, Warner JL, Wang L, Jiang M, Xu J, Chen Q, Nian H, Dai Q, Du X, Yang P, Denny JC, Liu H, Xu H. Discovery of noncancer drug effects on survival in electronic health records of patients with cancer: a new paradigm for drug repurposing. JCO Clin Cancer Inform. 2019;3:1-9.

14. Xu H, Aldrich MC, Chen Q, Liu H, Peterson NB, Dai Q, Levy M, Shah A, Han X, Ruan X, Jiang M, Li Y, Julien JS, Warner J, Friedman C, Roden DM, Denny JC. Validating drug repurposing signals using electronic health records: a case study of metformin associated with reduced cancer mortality. J Am Med Inform Assoc. 2015;22:179-91.

15. Garrison LP Jr, Neumann PJ, Erickson P, Marshall D, Mullins CD. Using real-world data for coverage and payment decisions: the ISPOR real-world data task force report. Value Health. 2007:10:326-35.

16. Health Level Seven International (HL7). HL7 FHIR implementation guide: minimal common oncology data elements (mCODE) release 1. 2019. http://hl7.org/fhir/us/mcode/2019Sep/. Accessed 16 Oct 2020.

17. American Society of Clinical Oncology. mCODE ${ }^{\mathrm{TM}}$ : Minimal Common Oncology Data Elements. 2020. https://mcodeinitiative.org/. Accessed 16 Oct 2020.

18. Ministry of Health, Consumer Affairs and Social Welfare. HCDSNS system: Digital Clinical History of National System of Health. Status report, October 1, 2020. https://www.mscbs.gob. es/profesionales/hcdsns/contenidoDoc/Inf_sit_HCDSNS_octub re_2020.pdf. Accessed 5 Mar 2021.

19. Sandoval MB, Val Palumbo M, Hart V. Electronic health record's effects on the outpatient office visit and clinical education. BMJ Health Care Inform. 2016;23:765.

20. Alkureishi MA, Lee WW, Lyons M, Press VG, Imam S, NkansahAmankra A, Werner D, Arora VM. Impact of electronic medical record use on the patient-doctor relationship and communication: a systematic review. J Gen Intern Med. 2016;31:548-60.

21. Bae J, Encinosa WE. National estimates of the impact of electronic health records on the workload of primary care physicians. BMC Health Serv Res. 2016;16:172.

22. Mazur LM, Mosaly PR, Moore C, Marks L. Association of the usability of electronic health records with cognitive workload and performance levels among physicians. JAMA Netw Open. 2019;2:e191709.

23. Kanas G, Morimoto L, Mowat F, O'Malley C, Fryzek J, Nordyke R. Use of electronic medical records in oncology outcomes research. Clinicoecon Outcomes Res. 2010;2:1-14.

24. Fasola G, Macerelli M, Follador A, Rihawi K, Aprile G, Della MV. Health information technology in oncology practice: a literature review. Cancer Inform. 2014;13:131-9.

25. Booth CM, Karim S, Mackillop WJ. Real-world data: towards achieving the achievable in cancer care. Nat Rev Clin Oncol. 2019;16:312-25.

Publisher's Note Springer Nature remains neutral with regard to jurisdictional claims in published maps and institutional affiliations. 


\section{Authors and Affiliations}

\section{N. Ribelles ${ }^{1}$ - I. Alvarez-Lopez ${ }^{2}$ - A. Arcusa ${ }^{3}$. J. I. Chacon ${ }^{4}$ - J. de la Haba ${ }^{5}$. J. García-Corbacho ${ }^{6}$ - J. Garcia-Mata ${ }^{7}$.} C. Jara ${ }^{8} \cdot$ J. M. Jerez ${ }^{9} \cdot$ M. Lázaro-Quintela ${ }^{10} \cdot$ L. Leon-Mateos ${ }^{11} \cdot$ N. Ramirez-Merino ${ }^{12} \cdot$ A. Tibau $^{13} \cdot$ A. Garcia-Palomo ${ }^{14}$

1 Medical Oncology Intercenter Unit, Regional and Virgen de la Victoria University Hospitals, IBIMA, Hospital Universitario Virgen de la Victoria, Campus Teatinos s/n., 29010 Málaga, Spain

2 Medical Oncology Department, Hospital Universitario Donostia-BioDonostia, Donostia, Gipuzkoa, Spain

3 Oncology Department, Consorci Sanitari de Terrassa, Barcelona, Spain

4 Medical Oncology Department, Hospital Virgen de la Salud, Toledo, Spain

5 Medical Oncology Department, Hospital Universitario Reina Sofía, Instituto de Investigación Biomédica de Córdoba (IMIBIC), Córdoba, Spain

6 Medical Oncology Department, Hospital Clinic/IDIBAPs, Barcelona, Spain

7 Medical Oncology Department, Complexo Hospitalario Universitario de Ourense, Ourense, Spain
8 Oncology Department, Hospital Universitario Fundación Alcorcón, Madrid, Spain

9 Department of Languages and Computer Science, E.T.S.I. Ingenería Informática, University of Málaga, Málaga, Spain

10 Medical Oncology Department, Hospital Álvaro Cunqueiro, Vigo, Spain

11 Medical Oncology Department, Complexo Hospitalario Universitario de Santiago de Compostela, Health Research Institute of Santiago (IDIS), Santiago de Compostela, Spain

12 Oncología Médica, Hospital Universitario Infanta Elena, Madrid, Spain

13 Medical Oncology Department, Hospital de la Santa Creu i Sant Pau, Barcelona, Spain

14 Medical Oncology Department, Complejo Asistencial Universitario de León, Instituto de Biomedicina (IBIOMED), León, Spain 